\title{
Public health in Thailand: emerging focus on non-communicable diseases
}

\author{
Nicholas D. Kaufman ${ }^{1 *}$, Sanchai Chasombat ${ }^{2}$, \\ Saengchom Tanomsingh ${ }^{3}$, Benjaporn Rajataramya ${ }^{1}$ \\ and Kathleen Potempa \\ ${ }^{1}$ Praboromarajchanok Institute, Ministry of Public Health, Thailand \\ ${ }^{2}$ Bureau of AIDS, TB and STI, Ministry of Public Health, Thailand \\ ${ }^{3}$ College of Public Health Administration, Ministry of Public Health, Thailand \\ ${ }^{4}$ University of Michigan, Ann Arbor, Michigan, USA
}

\section{SUMMARY}

Over the past three decades, the public health landscape in Thailand has shifted remarkably. Currently chronic non-communicable diseases represent the largest cause of mortality in the Thai population. In light of the current situation, this paper synthesizes what is known about the chronic non-communicable disease situation in Thailand and analyzes current policy responses. Relevant contextual factors such as socio-economic transitions, health systems development, and health workforce capacities are also considered. Primary data for this study were collected by a review of policy documents, government statements, and statistics reported by the Thailand Ministry of Public Health. Secondary data were obtained by a thorough review of the existing literature. The paper finds that while current policy responses to chronic non-communicable diseases in the health sector have focused on improving prevention and control of risk factors, a stronger emphasis on chronic disease treatment and management may be needed in the future. The paper concludes with an exploration of the potential for developing and implementing realistic public health responses to the growing burden of chronic non-communicable diseases in a Southeast Asian country context by utilizing existing capacities in research, policy, and health workforce development. Copyright (C) 2011 John Wiley \& Sons, Ltd.

KEY WORDS: Thailand; public health; cancer; cardiovascular disease; health policy

\section{INTRODUCTION}

Over the past three decades, the public health landscape in Thailand has shifted remarkably, as previously significant health concerns have been successfully addressed while new and re-emerging health problems have appeared. These shifts accompany changes in Thai society related to the dynamics of globalization

\footnotetext{
* Correspondence to: N. D. Kaufman, e-mail: ndkaufman@gmail.com
}

Copyright (C) 2011 John Wiley \& Sons, Ltd. 
and rapid economic growth, as well as the development of the Thai health care system.

Significant improvements have been achieved in the public health situation in Thailand. These include increasing life expectancy at birth from 55.9 years to 69.9 years for males and 62.0 years to 77.6 years for females from 1964 to 2006 (Rukumnuaykit, 2006). During this period the infant mortality rate (IMR per 1000 live births) declined from 84.3 to 11.3 , and the maternal mortality rate (MMR per 100000 live births) declined from 317.6 to 9.8 (Thailand Health Profile 2005-2007, 2008). Many previously important public health problems have been successfully addressed and are now on the decline, including some communicable diseases, malnutrition, maternal and perinatal mortality, and vaccine-preventable diseases. For example, from 1977 to 2006 the mortality rate from malaria has declined steadily from 10.7 per 100000 persons to 0.3 per 100000 persons, and the incidence rate has dropped from 7.9 per 100000 persons to 0.48 per 100000 persons (Thailand Health Profile 2005-2007, 2008). The incidence rate of encephalitis has dropped from 4.0 per 100000 and a mortality rate of 0.97 per 100000 persons in 1977, to 0.44 per 100000 and 0.02 per 100 000, respectively, in 2006 (Thailand Health Profile 20052007, 2008). HIV/AIDS, which is still a major health problem in Thailand, has been the focus of strong multi-sectoral efforts to implement prevention, treatment, and care programs since 1991 (Chasombat et al., 2006).

Given these developments and the increase in life expectancy, burden of disease in Thailand is increasingly shifting to non-communicable diseases (NCDs) such as cardiovascular diseases, cancers, and diabetes, as well as injuries and mental health disorders (WHO, 2009a). In 2002, the WHO Thailand Country Office estimated that of about 419000 deaths in Thailand, communicable, maternal and perinatal, and nutritional deficiencies accounted for $30 \%$, injuries accounted for $11 \%$, and chronic diseases accounted for the remaining 59\%. In light of the current situation, this paper provides a review of what is known about the chronic disease situation in Thailand, and assesses contextual factors such as socio-economic transition and health system development. The paper concludes by exploring the potential for developing and adapting public health responses that utilize existing capacities in research, policy, and health workforce development sectors.

\section{BACKGROUND}

\section{Socio-economic contexts in Thailand}

Thailand has experienced rapid economic development in recent years, with an economic boom beginning in 1989. During this period Thailand's Gross Domestic Product (GDP) rose from US\$ 50.5 billion in 1987 to US\$245.7 billion in 2007. Based on most recent World Bank data, Thailand has a population of 63.8 million and a gross national income per population of US\$ 3400 (World Bank, 2008). Thailand ranked \#78 out of 176 countries in 2006 in the United Nations Development Program's (UNDP's) Human Development Index (HDI) with a score of 0.786 , placing Thailand near the top of those countries considered to have a 'medium' level of human development (UNDP, 2008). 
Despite steady improvements in both HDI indicators and health, Thailand continues to be a country divided by poverty, and with regional variations in health status and health outcomes. Along with other parts of Asia, in recent years Thailand has experienced important trends in both urbanization and the aging of its population which are associated with epidemiological transition and particularly with risk factors for cardiovascular diseases (Yusef et al., 2001).

\section{Development of the Thai health care system}

Thailand's economic growth has enabled significant investment in its health care system. The Ministry of Public Health (MoPH) budget increased from 986.6 million baht in 1972 to 129.7 billion baht in 2007, with the most significant recent achievement being the establishment of a universal health care coverage policy in 2002 (Universal Coverage). As a proportion of GDP, total health expenditures in 2001 amounted to $3.2 \%$, and it is estimated that total national health expenditures will increase to $3.64 \%$ of GDP by 2010 and $3.88 \%$ of GDP by 2020 (Patcharanarumol et al., 2006). This compares with health expenditures as a proportion of GDP of $10.7 \%$ in Canada in 2008 (Canadian Institute for Health Information, 2008), and expenditures of $8.8 \%$ in Australia, 3.7\% in Singapore, 3.8\% in Malaysia, and 3.4\% in the Philippines in 2005 (Australian Institute of Health and Welfare, 2005). As a proportion of the national budget, the MoPH budget increased from 4.8\% in 1990 to 8.3\% in 2007 (Thailand Health Profile 2005-2007, 2008).

Improvements in healthcare financing have led to significant health systems development in Thailand. For example, medical equipment and health personnel have been distributed to rural health centers, (Putthasri et al., 2004) and quality assurance systems and drug procurement protocols established (Pongpirul, 2002). Yet significant regional discrepancies persist; for example a recent study of access to mammogram machines found for every one million females over 35 years old, 41.8 mammogram facilities were available in Bangkok, but only 2.8 facilities were available in the North and 4.0 in the Northeast (Putthasri et al., 2004). There have also been significant efforts to improve and expand the health workforce through recruitment and retention support. For example, the Praboromarajchanok Institute of Health Workforce Development has been responsible for the production of health personnel to fulfill MoPH needs since 1993, and currently operates 38 colleges across Thailand (Wongkongkathep et al., 2004; Praboromarajchanok Institute, 2007).

Thailand's system for the production and development of public health personnel has historically faced challenges in three areas: (1) policy and operation coordination with broader public health strategies at the national level; (2) building up equity in the availability of care and (3) reducing the problem of medical 'brain-drain' and personnel shortages in rural areas (Thailand Health Profile 2005-2007, 2008). The development of a professional nursing workforce, distributed throughout the country to the MoPH's lowest administrative unit (the sub-district or tambon of 10000 people) has been a particularly important component of efforts to overcome these challenges. There are currently 74 colleges/institutions in Thailand producing professional nurses, including 64 public and 10 private institutions (Thailand Health 
Profile 2005-2007, 2008). Of the Prabormarajchoanok Institute for Health Workforce Development's 38 colleges, 29 are nursing colleges located in different geographic regions across Thailand (Praboromarajchanok Institute, 2007).

\section{Chronic disease trends in the Asia-Pacific region}

Chronic non-communicable diseases such as heart disease, stroke, cancer, chronic respiratory diseases and diabetes are by far the most significant causes of mortality in the world, and $80 \%$ of chronic disease deaths occur in low and middle income countries (WHO, 2009b). In recent decades cardiovascular diseases have emerged as a leading cause of death in Asia, and across the South-East Asian region in particular (Asia Pacific Cohort Studies Collaboration, 2007). This may be linked to changing lifestyles and urbanization accompanying rapid economic development in some parts of the region as well as increase in life expectancy. Significant changes in diet, particularly increased consumption of fat and sugar, along with reductions in physical activity have occurred in many Asian countries (Popkin, 2001). High cholesterol is a known risk factor for many chronic diseases, yet awareness about the importance of raised cholesterol is relatively low among health care professionals, policy makers, and the general public in many Asian countries (WHO, 2002).

About half of the world's cardiovascular disease cases now occur in the AsiaPacific region. Smoking and increased levels of blood pressure are known to substantially increase the risk of cardiovascular diseases in Asian populations (Nakamura et al., 2008). The WHO predicts nearly half of the 89 million NCDrelated deaths projected for the South East Asian region over the next 10 years will occur prematurely, negatively impacting the social and economic development status of countries in the region (WHO, 2009c). Yet despite expectations that Asian countries will be among those countries experiencing the greatest increases in cardiovascular diseases over the next few decades, high-quality country-specific information about emerging epidemiological trends in NCDs remain low, and national research infrastructures are limited (InterASIA Collaborative Group, 2003).

\section{METHODS}

Primary data for this study derived from a review of MoPH policy documents, annual reports, and interpretation of Thailand government statistics. Information was collected from several MoPH departments, including the Bureau of NonCommunicable Diseases, the Bureau of Policy and Strategy, and the Praboromarajchanok Institute for Health Workforce Development. Policy and planning documents were translated from the Thai by the research team. Information was also gathered from interviews conducted with senior health officials in the Ministry of Public Health. A thorough review of existing academic literature was undertaken to capture the major public health challenges facing Thailand. Initial findings were integrated back into the investigation, and were used to guide further exploration and analysis of key issues as they emerged. The study methodology faced some 
limitations derived from the fragmented nature of nationally representative public health data in Thailand.

\section{RESULTS}

\section{Chronic disease trends in Thailand}

Cardiovascular diseases, cancers, chronic respiratory diseases, and diabetes are among the most significant emerging health concerns in Thailand, yet are often not detected until they have progressed to an advanced level. Nation-wide surveillance data on NCDs remain unreliable, though the MoPH has reported hospitalization data on morbidity (excluding Bangkok) from NCDs steadily rising from 1032 per 100000 population in 2001, to 1674 per 100000 population in 2004 (Bureau of Policy and Strategy, 2009b). As a result of excluding Bangkok, the largest urban area, these figures likely understate the true morbidity rate. In 2003, the InterAsia Collaborative Group reported all major risk factors for chronic diseases, except smoking, are higher in urban compared with rural areas in Thailand (InterASIA Collaborative Group, 2003).

A comparison of mortality statistics reported by the Bureau of Policy and Strategy between the years 1977 and 2008 (Bureau of Policy and Strategy Website, 2009a) illustrates the significant shifts in leading causes of death in recent years (see Table 1). We found reported death rates from non-communicable diseases have increased dramatically. While this increase could partly result from improvements in the quality of data collection and reporting on death rates and causes, this nonetheless suggests an increase in trend in a range of chronic non-communicable diseases.

This trend is well supported by data from other epidemiological studies. The 2006 Health and Welfare Survey of the Population and Social Statistics Group, Thailand National Statistics Office, reports $16 \%$ of the total population of Thailand has some form of chronic disease, with an average reported level of $35.6 \%$ among those over 45 years of age (Economic and Social Statistics Bureau, 2006). While this number reflects all chronic diseases, cardiovascular diseases accounted for $27.7 \%$ of those reported, and respiratory system diseases accounted for $8.9 \%$. A cohort study of employees of the Electricity Generating Authority of Thailand (EGAT) from 1985 to 1997 found several risk factors determine the chances of developing a noncommunicable chronic disease in Thailand, including age, sex, body mass index, waistline, hypertension, family history of diabetes, impaired glucose tolerance, triglyceride level, and HDL-cholesterol level. Importantly, the study found that some of the risk factors for developing chronic diseases in the Thai population can be controlled or modified (Sritara et al., 2003).

\section{Cardiovascular diseases}

In 2002, cardiovascular diseases were the single largest contributor to mortality in Thailand, estimated by the WHO to represent $20 \%$ of all death (WHO, 2009a). The WHO projects the prevalence of overweight individuals in Thailand to increase in 


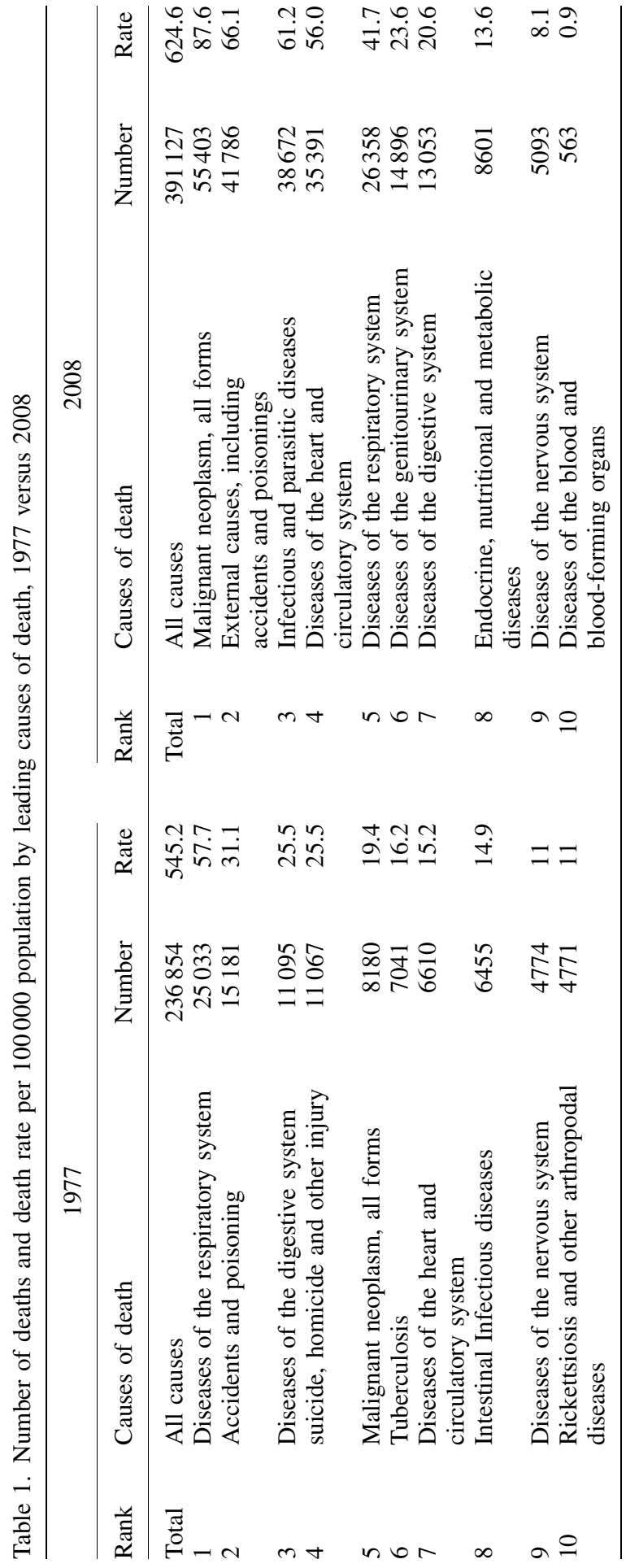


males and females aged 30 years and over from $34 \%$ to $35 \%$, and from $47 \%$ to $57 \%$, respectively, during the period 2005-2015 (WHO, 2009a). Obesity, defined by raised body mass index, is well known to be associated with increased incidence of cardiovascular diseases, type 2 diabetes, hypertension, stroke, dyslipidemia, osteoarthritis, and some types of cancers (Must et al., 1999).

Several large studies document the growing importance of cardiovascular diseases in Thailand. The Asia Pacific Cohort Study investigated the relationship between high total cholesterol (TC), defined as $\geq 6.2 \mathrm{mmol} / \mathrm{l}$, and the proportion of attributable fatal coronary heart disease and ischemic events that, theoretically, could be avoided if high TC were to be eliminated in 16 Asia-Pacific countries. They reported a population attributable fraction of $34 \%$ and $35 \%$ for males and females, respectively, in Thailand, higher than the Philippines (7\%, 12\%), Malaysia (21\%, $20 \%)$, China $(28 \%, 26 \%)$, and Taiwan $(32 \%, 26 \%)$, but lower than Singapore $(37 \%$, $31 \%)$, Japan $(36 \%, 34 \%)$, Australia $(46 \%, 41 \%)$, and New Zealand. (47\%, 42\%) (Woodward et al., 2008). A study of cardiovascular risk levels in urban and rural Thailand involving a structured questionnaire, brief physical examination and a blood sample from 5305 participants aged 35 years and older found absolute levels of risk factors to be high, with all major risk factors except smoking worse in urban rather than rural areas (InterASIA Collaborative Group, 2003). This study, conducted with data collected in 2000, estimated the following population prevalence of major risk factors in individuals aged 35 years and older (see Table 2). Chronic obstructive pulmonary disease is projected to trend steadily upwards, with estimates per 100000 population rising from 2268 in 2001 to 7035 in 2010 (Thailand Public Health Profile 2001-2004, 2005).

In 2006, the MoPH Bureau of Epidemiology reported 792035 cases of hypertension, of which the majority were female, 501625, while 290428 were male (Annual Epidemiological Surveillance Report, 2006). In the same year 83047 cases of ischemic heart disease were reported, with 40531 male patients and 42516 female patients (Annual Epidemiological Surveillance Report, 2006). Comparing this with subsequent data from the 2007 Annual Epidemiological Surveillance Report suggests an upward trend in each disease, with 1047154 cases of hypertension (389 834 male, 657319 female); and 121729 cases of ischemic heart disease (58 079 male, 63650 female) occurring in Thailand (Annual Epidemiological Surveillance Report, 2007).

Table 2. Major risk factors in Thailand

\begin{tabular}{lccc}
\hline Risk factor & $\begin{array}{c}\text { Prevalence } \\
\text { in Thai } \\
\text { Males } \geq 35 \text { years }\end{array}$ & $\begin{array}{c}\text { Prevalence } \\
\text { in Thai } \\
\text { Females } \geq 35 \text { years }\end{array}$ & Total $\geq 35$ years \\
\hline High blood pressure & $21 \%$ & $20 \%$ & $21 \%$ \\
High cholesterol & $14 \%$ & $21 \%$ & $18 \%$ \\
Diabetes & $9 \%$ & $10 \%$ & $9.6 \%$ \\
Overweight & $28 \%$ & $43 \%$ & $36 \%$ \\
Current cigarette smoker & $48 \%$ & $3 \%$ & $25 \%$ \\
\hline
\end{tabular}


Cancer

Cancer is a significant concern in Thailand, representing 14\% of all deaths in 2002. There is a troubling trend as the hospital admission rate for cancer per 100000 population has increased from 34.7 in 1994 to 124.4 in 2006 (Thailand Health Profile 2005-2007, 2008). In comparison with other countries with developed health care systems such as the US, the level of screening and early diagnosis in Thailand is low. For example, a 2004 health examination survey of females aged 15-59 found just $49 \%$ of respondents had ever been screened for cervical cancer, $23-24 \%$ had ever received a breast examination from a health care professional, and $4 \%$ had ever received a mammogram (Thailand Health Profile 2005-2007, 2008). According to the National Cancer Institute, the highest rate of breast cancer has been found in the Bangkok Metropolitan area, and the Thanyarak Breast Cancer Treatment Center at Siriraj Hospital, one of the largest teaching hospitals in Thailand, reported $80 \%$ of Thai female breast cancer patients were at invasive stages of progression (Thailand Health Profile 2005-2007, 2008). Of the Thanyarak Center's 5994 patients between 1995 and 2004, 2487 or $41.5 \%$ were between the ages of 40 and 49 (Thailand Health Profile 2005-2007, 2008). Most recent data from 2008 show of 795 new breast cancer patients, $422(53.1 \%)$ were between the ages 40 and 55 (National Cancer Institute, 2009). This contrasts with data from the American Cancer Society (2006), which report the largest proportion of US breast cancer patients are females aged 7079 (24.2\% of all patients), while just $5.1 \%$ of patients were less than 40 years of age. A recent cohort study of breast cancer risk factors in Thailand suggest substantial increases in breast cancer rates in Thailand could be expected in the future (Jordan et al., 2009).

Liver cancer is a significant concern in Thailand, with Vatanasapt and Sriamporn (1999) reporting that Khon Kaen province, Thailand, has the highest incidence of liver cancer in the world in females (32.7 per 100000 population), and an extremely high incidence rate in males ( 85.0 per 100000$)$. Overall the national incidence rate of liver cancer per 100000 population was 31.2 in males and 11.5 in females in 2000. Most recent data from the National Cancer Institute of Thailand (2009) report the most common cancers in 2008 in males were: colon and rectum $(17.4 \%)$, bronchus and lung $(16.2 \%)$, liver and bile duct $(10.5 \%)$, oral cavity $(8.5 \%)$, esophagus $(5.1 \%)$, nasopharynx (4.4\%), and non-Hodgkin lymphoma (4.1\%). In women, most common cancers in 2008 were breast $(43 \%)$, cervix and uteri $(16.4 \%)$, colon and rectum $(8.8 \%)$, bronchus and lung $(5.2 \%)$, liver and bile duct $(3.3 \%)$, corpus uteri $(3.1 \%)$, and oral cavity $(2.9 \%)$.

\section{HIV/AIDS-related cancer}

The Bureau of AIDS, TB and STIs at the Thailand MoPH (2008) provide updated estimates for the Asian Epidemic Model (AEM) Projections for HIV/AIDS in Thailand 2005-2025, estimating that in 2008 there are 1115000 persons who have been infected with HIV/AIDS since the start of the epidemic, of which 532500 people are still alive and currently living with HIV. People with immune deficiency from HIV have been found to be at increased risk of numerous cancer types. 
However, in the Thai context little is known about the relative risk cancer in HIVpositive people. Much of the limited research on HIV/AIDS related cancer has used large clinical cohorts located in North American or European settings, and very little is known about the situation for HIV/AIDS patients in low and middle income country settings where highly active antiretroviral therapy (HAART) have been widely rolled out in recent years.

A 1998 US study of data from 98336 people with AIDS and 1125098 people with cancer found AIDS patients have increased risk of numerous types of cancer compared with the general population, including lip, oropharyngeal, laryngeal, stomach, liver, Hodgkin's disease, lymphoid leukemia, multiple myeloma, seminoma, anal, melanoma, non-melanoma skin, and lung cancers (Goedert et al., 1998). A recent meta-analysis by Grulich et al. (2007) compared large population-based cohort studies of people with HIV/AIDS and people immunosuppressed after solid organ transplantation to determine whether a wider range of cancers is associated with immune-deficient status. Their findings indicate immune deficiency is responsible for increased risk of cancer, and suggests infection-related cancer will become an increasingly important complication of long-term HIV infection. This has potentially important implications for people on HAART in Thailand, and suggests detection and treatment of cancers could become an increasingly important component of the HIV care landscape. However, the resources available for prevention, diagnosis and treatment of cancers in Thailand remain limited, and interactions between available ART regimens, differing social, behavioral, and environmental risk factors, and the profile of cancers of HIV patients remain poorly understood. Moreover, there are significant gaps in knowledge about HIV-related cancers to be filled with research done in the Thai context.

\section{Obesity and diabetes}

Obesity and overweight are known to be major risk factors for chronic diseases including type 2 diabetes, cardiovascular disease, hypertension and stroke, and certain forms of cancer globally (WHO, 2003a). A 2002 study of transitions in nutrition and dietary patterns in Thailand found strong evidence of increasing trends in obesity and overweight over the past three decades (Kosulwat, 2002). Obesity and overweight were found to be a major risk factor for coronary heart disease in a cohort study of 2536 male employees of the Electricity Generating Authority of Thailand at 17 years follow-up (Aekplakorn et al., 2007). Upward trends in obesity rates among Thai children have been described with concern by the International Health Policy Program of the MoPH (Pulkerd, 2007), as well as by the WHO which noted the prevalence of obesity in 5-12-year old children in Thailand rose from $12.2 \%$ to 15 $6 \%$ in just 2 years (WHO, 2003b). In 2006, the Bureau of Epidemiology identified 609654 cases of diabetes in the Thai population, of which 201699 were male while 407955 were female (Annual Epidemiological Surveillance Report, 2006). The following year 714892 diabetes cases (229715 male, 485177 female) were reported, representing an increase of $17.26 \%$ (Annual Epidemiological Surveillance Report, 2007). While the increase in total number of diagnosed cases of diabetes may be 
related to advances in screening and diagnostic capabilities and access to medical care, the upward trend is likely also related to nutrition.

\section{Kidney disease}

Chronic kidney disease is a significant concern in Thailand (Narenpitak and Narenpitak, 2008) with 12884 deaths and a death rate of 20.6 per 100000 persons occurring from nephritis, nephrotic syndrome and nephrosis in 2006 (Annual Epidemiological Surveillance Report, 2006). A recent study of the prevalence of stage III and IV chronic kidney disease in Thailand was conducted in 2008 using a stratified, multistage, cluster-sampling method of individuals aged 35 and above from the Thai 2000 Census (Perkovic et al., 2008). The study found the prevalence of stage III kidney disease to be about $20 \%$ using a Cockroft-Gault formula and about $13 \%$ using a simplified Modification of Diet in Renal Disease formula. Using these respective formulas, the study found prevalence of stage IV kidney disease to be about $0.9 \%$ and $0.6 \%$ of this population. The prevalence of both stage III and IV chronic kidney disease was significantly higher than reported in individuals over 40 years old from the United States, and also higher than the reported incidence in Taiwan and Australia (Narenpitak and Narenpitak, 2008).

\section{Thailand's response to chronic non-communicable diseases}

The Thai health care authorities have recognized the burden of NCDs, and the MoPH is working to address this problem. The Bureau of Non-communicable Diseases, in the Department of Disease Control, MoPH, has primary responsibility for NCD prevention and control in Thailand. The Bureau was established in 2003; previously NCDs had been addressed through Thailand's National Health Development Plan (2002-2004). Over the past 10 years, the MoPH has undertaken a series of crosscutting programs to address major risk factors, including a Comprehensive Tobacco Control Program, Comprehensive Alcohol Control Program, several nutrition related programs (Clean Food Good Taste, dietary guidelines and education, healthy recipes, nutritional labeling for NCD risk reduction, 'Children Not to Eat Sweet' network), and interventions promoting physical activity (Empowerment for Health, Exercise for Health) (Supornsilaphachai, 2005). Additional areas in which NCD risk prevention and control activities have been pursued include:

- cervix screening and self breast examination promotion,

- cardiovascular diseases, hypertension and diabetes awareness programs,

- quality improvement for hypertension and diabetes screening,

- health promotion in schools since 1999,

- Jump Rope Project initiated by Thai Heart Foundation,

- Healthy Work Place program since 2000,

- Health Promoting Hospital since 2000,

- Healthy Cities initiative since 1999.

In Thailand, the current guiding document which serves as a position statement for national NCD priorities is the Bureau of NCDs Strategic Planning Framework 2008- 
2011 (Bureau of Non-Communicable Diseases, 2008). This framework outlines seven principles that are intended to direct the national policy and programming activities of the Bureau. These are: (1) innovation in responding to NCDs; (2) human resources development; (3) establishing national policies and activities to support local-level NCD prevention, surveillance, and control; (4) developing surveillance systems and reliable data collection and management; (5) strengthening networks among hospitals and health care workers; (6) changing attitudes, values, and organizational culture within the health care system; (7) developing resource management and monitoring and evaluation capacities (Bureau of Non-Communicable Diseases, 2008).

To coordinate NCD prevention and control activities at the national level the $\mathrm{MoPH}$ has also established the Partnerships for Prevention and Control of NCDs Working Group (Bureau of Non-Communicable Diseases, 2009). This working group is intended to link the Bureau of Non-Communicable Diseases with the other relevant departments throughout the $\mathrm{MoPH}$, including the Department of Health (responsible for family health and health promotion), the Department of Health Services Support, the Department of Thai Traditional Medicine, the Department of Mental Health, the Bureau of Policy and Planning, the Food and Drug Administration, and the National Health Security Office. The working group objectives are to achieve broad behavior changes in the general population and highrisk groups, and improved social, environmental, health, and economic status, leading to healthier Thai citizens. The working group is currently implementing six action plans: (1) surveillance and screening of high risk groups for diabetes at both community hospitals and using local health volunteers; (2) awareness and education about NCDs and a national behavior change campaign; (3) improving environmental conditions and risk reduction in communities; (4) counseling and behavior change in high risk groups and NCD patients; (5) capacity building for health workers in NCD prevention, and health systems strengthening; (6) monitoring and evaluation of all activities (Bureau of Non-Communicable Diseases, 2009). An important feature of this working group is its mandate to promote NCD responses that are geared to Thai cultural and traditional contexts, including prevention of chronic diseases at the family and community level. For example, the project 'Health with Thai Lifestyle' is an innovative health promotion project led by the Bureau of NCDS that engages community health volunteers to act as role models for healthy living within Thai lifestyles, and to provide advice promoting healthy living in communities throughout Thailand.

The development of existing national policy and programming frameworks for NCD prevention and control in Thailand have been guided by a comprehensive SWOT (Strengths, Weaknesses, Opportunities, Threats) analysis of the NCD situation and relevant MoPH capabilities, conducted by the Department of Disease Control (Bureau of Non-Communicable Diseases, 2008). This analysis found key strengths include: the existence of capable staff and competent healthcare workers throughout Thailand, the presence of academic experts connected nationally and internationally, effective senior level management, strong policy development processes at the national level, and the experience of having successfully implemented the a Comprehensive Tobacco Control Program. Weaknesses include: 
unorganized local and mid-level management, staff shortages and high workloads in rural areas, little time or opportunity for continuing training, weak resource management, and unclear treatment guidelines for some NCDs. Key opportunities were seen to include a strong national policy response to NCDs, capable networks of health care workers and hospitals, a Department of Disease Control administrative center located in every region of Thailand, budget support from both public and private sectors, and support from academics and research. Main threats were identified as the negative influence of the media and advertising leading to changes in lifestyles and increasing risks for NCDs, inconsistent and unreliable information about NCDs are being distributed to the public, low public awareness of NCD issues especially among less well educated people, and high healthcare worker turnover in rural areas (Bureau of Non-Communicable Diseases, 2008).

\section{DISCUSSION}

In light of the emerging focus on chronic non-communicable diseases in Thailand's public health landscape, the need for national health policies and systems to be in step with population needs is critical. The increasing pressure that NCDs are placing on the Thai health care system is clearly illustrated by the increase in hospitalization rates between 1985 and 2006. Between 1985 and 2006, the rate of hospitalization of patients per 100000 population for heart diseases grew from 63.4 to 618.5 , for cancer from 53.8 to 124.4, and for diabetes from 33.3 to 586.8 (Thailand Health Profile 2005-2007, 2008). Yet it is clear from current policy frameworks that the Thai health authorities' current approach towards NCDs prioritizes population level prevention, behavior change, and control, while considerably less emphasis is placed on expanding treatment and chronic disease management capacities within the health care system.

The importance of strong prevention programs is well supported by evidence from studies conducted in Europe and North America, which indicate that significant reductions in mortality from NCDs can be achieved with relatively simple public health interventions targeting modifiable risk factors such as blood pressure, blood cholesterol, smoking, obesity, and their socio-economic determinants at population, community and individual levels (Darnton-Hill et al., 2004; Nishida et al., 2004). Yet as more lives are prolonged or saved/spared from communicable diseases, there will be chronic conditions whose onset will be prolonged but not totally prevented, as trends in Western countries indicate (Cooper et al., 2000; Edward et al., 2005). Moreover, rapid upward trends in numerous risk factors portend far more substantial chronic disease treatment needs may be looming on the horizon for the Thai health care system. Given these factors, Thailand needs to ensure its health care system is prepared to meet the emerging and future challenges that NCDs will pose. Expanding NCD planning and policy frameworks to include a stronger focus on treatment and chronic disease management is recommended.

Public health policies in Thailand should focus on achieving appropriate selection and use of chronic care and treatments within the health system, affordable and sustainable chronic care management within the existing public health insurance 
schemes, and a health workforce sufficiently trained in managing chronic conditions and rationally distributed throughout all geographic regions. These are essential to ensuring equitable access to care and treatment, particularly in poorer and geographically remote regions in Thailand.

Positioning the Thai health care system to meet steadily increasing chronic care demands is a resource allocation decision, with significant implications for the way care is provided to people with chronic diseases. Thailand's current health financing cannot support a dramatic expansion of high-cost clinical procedures under Universal Coverage, so realistic approaches are needed that maximize Thailand's existing strengths and health services capacities. Thailand has achieved affordable and accessible care for certain types of patients requiring long-term care, for example AIDS patients, through a system of distributing stable patients from larger provincial hospitals back to more local community hospitals and primary care centers. This has required building AIDS care and management capacities throughout all levels of hospitals, and particularly within Thailand's professional nursing workforce. Similar efforts with Thailand's nursing workforce could provide added support for decentralized NCD care, and improve NCD treatment and management capacities at community hospitals and more local primary care treatment centers. Additional capacity building for community hospitals and health workforces could focus on areas such as chronic disease treatment and chronic care management, streamlined patient data storage and management, drug procurement and storage systems, and financial reporting and budgeting support.

The human resource needs for chronic care management are different from the human resource needs for prevention-based interventions, and will require adapting the human resource development strategy of the MoPH. Thailand's impressive professional nursing workforce, already well established with a ratio of nurse/ population of 1/619 in 2006, is distributed throughout all levels of the health care system in all geographic regions (Health Information Unit, Bureau of Policy and Strategy, 2006) and may be crucial to meeting the future chronic treatment needs of the Thai population. For example, Potempa et al. (2009) have shown that efforts to leverage capacities in nursing to provide care for HIV patients are possible and successful in the Thai context.

A stronger focus on strengthening the research capacities of the MoPH may also be needed to achieve the Bureau of NCDs stated policy directives of innovation in NCD responses, and improved surveillance and data management. For example, research on chronic disease management in the Thai context could potentially identify and promote ways of providing chronic care and treatment that are better geared to Thai cultural and community realities and socio-economic contexts.

\section{CONCLUSION}

This review of the state of chronic disease in Thailand is timely, and underscores the shifting trends in mortality currently under way. Cost effective interventions to prevent and treat emerging chronic diseases exist, and have worked in many other countries. Based on the trends described in this paper, we conclude similar 
interventions could work in Thailand, and are needed. Successful strategies elsewhere have involved multi-layered initiatives combining population-based approaches with interventions targeting individual behavior change/modification as well as chronic treatment management. This review indicates additional research is needed to better understand the epidemiology of non-communicable chronic diseases in Thailand, as well as to guide the development and implementation of interventions to improve treatment and management of chronic disease patients. Multi-sectoral efforts are necessary spanning concerted policy actions and legislative shifts, as well as interventions involving a diversity of delivery channels beyond just the MoPH, such as other relevant ministries, public opinion leaders, politicians, legislative bodies, and policy makers in various education and law enforcement systems.

\section{REFERENCES}

Aekplakorn W, Pakpeankitwatana V, Lee C, Woodward M, Barzi F, Yamwong S, Unkurapinun S, Sritara P. 2007. Abdominal obesity and coronary heart disease in Thai men. Obesity 15: 1036-1042.

American Cancer Society. 2006. Breast Cancer Facts and Figures 2005-2006. American Cancer Society, Inc.: Atlanta.

Asia Pacific Cohort Studies Collaboration. 2007. Cholesterol, diabetes and major cardiovascular diseases in the Asia-Pacific region. Diabetologia 50(11): 2289-2297.

Australian Institute of Health and Welfare. 2005. Health Expenditures Australia 2003-04. Health and Welfare Expenditure Series 2: Canberra, Australia.

Bureau of AIDS, TB and STIs. 2008. The Asian Epidemic Model (AEM) Projections for HIV/AIDS in Thailand: 2005-2025. Department of Disease Control, Ministry of Public Health: Thailand.

Bureau of Epidemiology. 2006. Annual Epidemiological Surveillance Report. Ministry of Public Health: Thailand.

Bureau of Epidemiology. 2007. Annual Epidemiological Surveillance Report. Ministry of Public Health: Thailand.

Bureau of Non-Communicable Diseases. 2008. Strategic Planning Framework, 4-Year Plan 20082011. Department of Disease Control, Ministry of Public Health: Thailand.

Bureau of Non-Communicable Diseases. 2009. Committee Meeting Report on Healthy Thai Living, Partnerships for Prevention and Control of NCDS Working Group, 10 November 2009, Department of Disease Control, Ministry of Public Health.

Bureau of Policy and Strategy. 2005. 2001-2004 Thailand Public Health Profile. Ministry of Public Health: Thailand.

Copyright (C) 2011 John Wiley \& Sons, Ltd.
Bureau of Policy and Strategy. 2006. Number and Proportion Between Nurse and Population Separated as Regional Attribute. Ministry of Public Health: Thailand; Available from URL: http://bps. ops.moph.go.th/webenglish/Resources/per 16-2006. xls [Accessed om 14 June 2009].

Bureau of Policy and Strategy. 2008. Thailand Health Profile 2005-2007. Ministry of Public Health: Thailand.

Bureau of Policy and Strategy Website. 2009a. Mortality Statistics, http://bps., ops., moph., go., th/webenglish/ Information.htm [Accessed June 2009].

Bureau of Policy and Strategy. 2009b. Number and Rate of In-patients According to 75 Groups of Causes from Health Service Units. Ministry of Public Health: Thailand Available from URL: http:// bps.ops.moph.go.th/webenglish/Information/ill-in 42-48. htm [Accessed on 10 June 2009].

Canadian Institute for Health Information. 2008. National health expenditure trends. Available from URL: http://secure.cihi.ca/cihiweb/dispPage. jsp?cw_page=media_13nov2008_e [Accessed 18 March 2009].

Chasombat S, Lertpiriyasuwat C, Thanprasertsuk S, Suebsaeng L, Lo YR. 2006. National access to antiretroviral program for PHA (NAPHA) in Thailand. Southeast Asian J Trop Med Public Health 37: 704-715.

Cooper R, Cutler J, Desvigne-Nickens P, et al. 2000. Trends and disparities in coronary heart disease, stroke, and other cardiovascular diseases in the United States: findings of the national conference on cardiovascular disease prevention. Circulation 102: 3137-3147.

Darnton-Hill I, Nishida C, James WPT. 2004. A life course approach to diet, nutrition and the prevention of chronic diseases. Pub Health Nutr 7(1A): 101121 .

Int J Health Plann Mgmt 2011; 26: e197-e212. DOI: $10.1002 / \mathrm{hpm}$ 
Economic and Social Statistics Bureau. 2006. Health and Welfare Survey 2006, hdl:10527/11053. National Statistics Office: Thailand.

Edward W, Gregg EW, Cheng YJ, et al. 2005. Secular trends in cardiovascular disease risk factors according to body mass index in US adults. JAMA 293 1868-1874.

Goedert JJ, Cote TR, Virgo P, et al. 1998. Spectrum of AIDS-associated malignant disorders. Lancet 351(9119): 1833-1839.

Grulich AE, van Leeuwen MT, Falster MO, Vajdic CM 2007. Incidence of cancers in people with HIV/AIDS compared with immunosuppressed transplant recipients: a meta-analysis. Lancet 370(9581): 59-67.

InterASIA Collaborative Group. 2003. Cardiovascular risk factor levels in urban and rural Thailand-The International Collaborative Study of Cardiovascular Disease in Asia (InterASIA). Eur J Cardiovasc Prev Rehabil 10(4): 249-257.

Jordan S, Lim L, Vilainerun D, et al. 2009. Breast cancer in the Thai Cohort Study: an exploratory case-control analysis. Breast 18(5): 299-303. Epub 2 Oct 2009.

Kosulwat V. 2002. The nutrition and health transition in Thailand. Pub Health Nutr 5(1A): 183-189.

Must A, Spadano J, Coakley EH, Field AE, Colditz G, Dietz WH. 1999. The disease burden associated with overweight and obesity. JAMA 282(16): 1523-1529.

Nakamura K, Barzi F, Lam TH, et al. 2008. Cigarette smoking, systolic blood pressure, and cardiovascular diseases in the Asia-Pacific region. Stroke 39(6): 1694-1702.

Narenpitak S, Narenpitak A. 2008. Prevalence of chronic kidney disease in type 2 diabetes in primary health care unit of Udon Thani Province, Thailand. $J$ Med Assoc Thailand 91(10): 1505-1513.

National Statistics Office of Thailand. 2007. The Health and Welfare Survey. Government of Thailand: Bangkok.

National Cancer Institute. 2009. Hostital-Based Cancer Registry. Attasara P., Buason R. (eds). 2008 Department of Medical Services, Ministry of Public Health: Thailand.

Nishida C, Uauy R, Kumanyika S, Shetty P. 2004. The Joint WHO/FAO Expert Consultation on diet, nutrition and the prevention of chronic diseases: process, product and policy implications. Pub Health Nutr 7(1A): 245-250

Patcharanarumol W, Cichon M, Tangcharoensathien V, Vasvid C, Tisayaticom K. 2006. Research Series of Thai Healthcare Financing: Part 1 financial reform options of healthcare coverage in Thailand. J Health Sci 15(1): 17-30.

Perkovic V, Cass A, Patel AA, et al. 2008. High prevalence of chronic kidney disease in Thailand. Kidney Int 73(4): 473-479.

Copyright (C) 2011 John Wiley \& Sons, Ltd.
Pongpirul K. 2002. Quality Assurance of Health Promotion Services in Provincial Health System. International Health Policy Program, Ministry of Public Health: Thailand.

Popkin BM. 2001. The nutrition transition and obesity in the developing world. J Nutr 131: 871S-873S

Potempa K, Phancharoenworakul K, Glass N, Chasombat S, Cody N. 2009. Leveraging the role of public health nursing in managing HIV/AIDS in Thailand: a journey of international collaboration. Collegian: J Royal College Nursing Australia 16(2): 49-53.

Praboromarajchanok Institute for Health Workforce Development. 2007. 2007 Annual Report. Ministry of Public Health: Thailand.

Pulkerd S. 2007. Obesity among school children in Thailand: inquiry on demand for, supply of fast food, healthy food, policy interventions. International Health Policy Program, Ministry of Public Health, Thailand. Available from URL: http://ihppthaigov., net/presentation/attachjournal/42/PPT/PPT1.,ppt\# 266,2,Outline [Accessed March 11, 2009].

Putthasri W, Tangcharoensathien V, Mugem S, Jindawatana W. 2004. Geographical Distribution and Utilization of Mammography in Thailand. Regional Health Forum 8:1 World Health Organization: South East Asia Regional Office.

Rukumnuaykit P. 2006. Mortality and causes of death in Thailand: evidence from the survey of population change and death registration. Asia-Pacific Popul J 21: 2 .

Sritara P, Cheepudomwit S, Chapman N, et al. 2003. Twelve-year changes in vascular risk factors and their associations with mortality in a cohort of 3499 Thais: the Electricity Generating Authority of Thailand Study. Int J Epidemiol 32(3): 468469

Supornsilaphachai C. 2005. National NCD Prevention and Control Policies, Programmes and Networks. Bureau of Non-Communicable Diseases, Ministry of Public Health: Thailand.

United Nations Development Program. 2008. Human Development Report 2008 Statistical Update. Country Fact Sheet Thailand. Available from URL: http://hdrstats.undp.org/2008/countries/country_fact_sheets/cty_fs_THA.html [Accessed March 11 2009].

Vatanasapt V, Sriamporn S. 1999. Liver cancer. In Cancer in Thailand Vol. II, 1992-1994. IARC Technical Report No. 34, Deerasamee S, Martin N, Sontipong S, Sriamporn S, Sriplung H, Srivatanakul P, Vatanasapt V, Parkin DM, Ferlay J (eds). IARC: Lyon; 45-48.

Wongkongkathep S, Hiranprueck A, Suwarnnata N, Yokakul S. 2004. Evaluation on organization

Int J Health Plann Mgmt 2011; 26: e197-e212. DOI: $10.1002 / \mathrm{hpm}$ 
development for excellence: a case study of the Praboromarajchanok Institute of Health Workforce Development. J Health Sci 13: 598607.

Woodward M, Martiniuk A, Ying Lee CM, et al. 2008. Elevated total cholesterol: its prevalence and population attributable fraction for mortality from coronary heart disease and ischaemic stroke in the Asia-Pacific region. Eur J Cardiovasc Prev Rehabil 15(4): 397-401.

World Bank. 2008. Country Statistical Information, Thailand at a Glance. World Bank: Bangkok, Thailand.

World Health Organization. 2002. Non-communicable diseases and mental health: integrated management of cardiovascular risk. Available from URL: http:// www.who.int/cardiovascular_diseases/media/en/ 635.pdf [May 12 2009].

World Health Organization. 2003a. Diet, nutrition and the prevention of chronic diseases: report of a joint
WHO/FAO expert consultation. Technical Report Series 916, Geneva

World Health Organization. 2003b. Global strategy on diet, physical activity and health fact sheet: obesity and overweight. Geneva

World Health Organization. 2009a. Facing the Facts: The Impact of Chronic Disease in Thailand. World Health Organization Thailand Country Office: Nonthaburi, Thailand.

World Health Organization. 2009b. Chronic diseases and health promotion. Available from URL: http:// www.who.int/chp/en/index.html [March 10 2009].

World Health Organization Website. 2009c. Available from: URL: http://w3.whothai.org/EN/Section3/ Section106.htm [March 10 2009].

Yusef S, Reddy S, Ounpuu S, Anand S. 2001. Global burden of cardiovascular diseases. Part 1: General considerations the epidemiological transition, risk factors, and impact of urbanization. Circulation 104: $2746-2753$. 